\title{
Utility of measuring fractional flow reserve by CT angiography in patients with suspected CAD
}

$\mathrm{P}$ atients presenting with stable chest pain are assessed using invasive or noninvasive measures to confirm

the presence or absence of coronary artery disease $(\mathrm{CAD})$ and to guide medical care. Although current guidelines recommend the use of noninvasive stress testing for these patients, the number of invasive angiograms showing no obstructive CAD remains high. Previous trials have indicated that coronary computed tomographic angiography (CTA) can improve diagnostic accuracy and efficiency of triage to catheterization. Since this strategy provided only anatomical data, the functional relevance of coronary stenoses identified using CTA was unclear. To address this limitation, a noninvasive method to calculate fractional flow reserve (FFR) by CTA $\left(\mathrm{FFR}_{\mathrm{CT}}\right.$ ) was developed. Investigators of the PLATFORM trial sought to determine whether CTA/ FFR $_{\mathrm{CT}}$-guided assessment of patients with suspected CAD would result in fewer invasive angiograms showing no obstructive CAD compared with patients undergoing standard assessment.

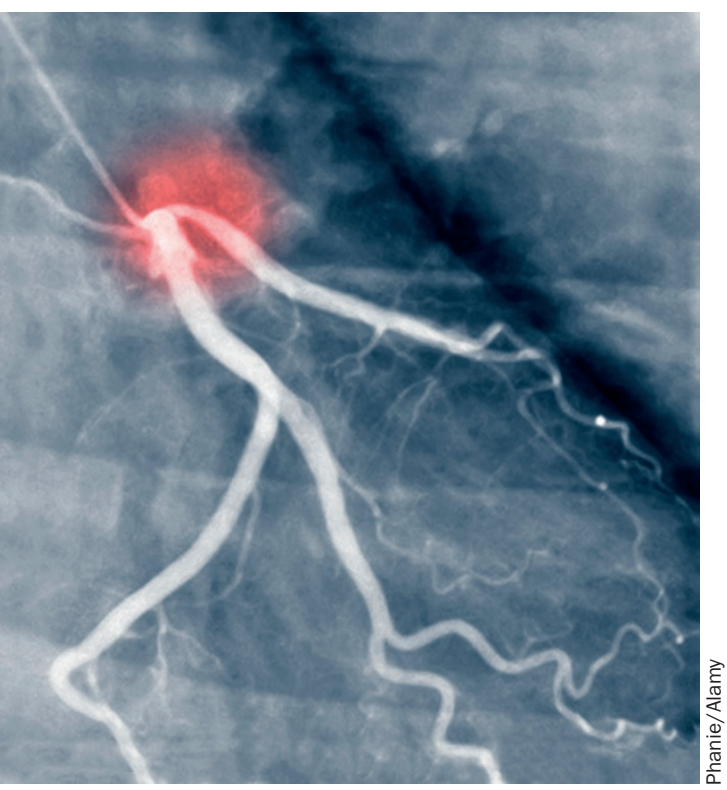

The findings in this study were presented at the ESC Congress 2015 and simultaneously published in the European Heart Journal.

The PLATFORM trial is a prospective, consecutive cohort study into which researchers enrolled patients with newonset chest pain and suspected CAD for which noninvasive or invasive assessment had been planned. Participants were assigned to receive either standard assessment or CTA/FFR ${ }_{\mathrm{CT}}$ testing. The primary end point was the rate of invasive coronary angiography (ICA) within 90 days that showed no evidence of obstructive CAD in patients with preplanned (that is, planned before enrolment) invasive testing. The secondary end point was the rate of ICA with no obstructive CAD in patients with preplanned noninvasive testing. The major safety end point was a composite of allcause death, myocardial infarction, and unplanned hospitalization for chest pain requiring revascularization at 90 days.

A total of 584 patients were enrolled into the study from 11 sites across Europe. Among 204 patients who had a preplanned noninvasive test, 104 were allocated to $\mathrm{CTA} / \mathrm{FFR}_{\mathrm{CT}}$ testing and the remainder were assigned to usual care. The rate of ICA that showed no obstructive CAD was not different in patients receiving usual care compared with patients in the CTA/ $\mathrm{FFR}_{\mathrm{CT}}$ testing group (6.0\% versus $12.5 \%$; $P=0.95)$. Among the 308 patients with preplanned invasive catheterization, 187 were assigned to usual care (ICA), and 193 patients were allocated to undergo $\mathrm{CTA} / \mathrm{FFR}_{\mathrm{CT}}$-guided testing. The primary end point, or the number of ICA cases which did not show obstructive CAD, occurred in $73.3 \%$ of patients in the usual-care group, compared with $12.4 \%$ in the CTA/FFR ${ }_{\mathrm{CT}}$ group (risk difference $60.8 \%, 95 \%$ CI 53.0-68.7, $P<0.0001$ ). Importantly, results were similar across all subgroups. Patients in these two assessment groups also had similar mean cumulative radiation exposure and rates of major adverse cardiovascular events. The rates of revascularization of patients assigned to CTA/FFR ${ }_{\mathrm{CT}}$-guided testing compared with usual care were also not different $(P=0.58)$. Use of $\mathrm{FFR}_{\mathrm{CT}}$ resulted in cancellation of $61 \%$ of ICAs and significantly increased the availability of functional information for patients proceeding to ICA and revascularization.

Pamela Douglas, the lead investigator of the PLATFORM trial, emphasizes that while "the technique of FFR $_{\mathrm{CT}}$ has been successfully validated against invasive FFR ... the impact of its use on clinical care processes and outcomes had not been previously evaluated". Given that CTA is not currently a class I recommendation in US or European guidelines, data from this study "add to the growing body of evidence that this testing option is a reasonable choice for further evaluation of patients with stable chest pain".

William F. Fearon from Stanford University Medical Center in Stanford, USA, who was not involved in the study, welcomes these positive findings and highlights the importance of future studies. "The next steps will be to look at the financial implications of this strategy," he explains. "If those results are favourable, this new approach could become the standard method for evaluating patients with suspected coronary disease." The PLATFORM investigators will be presenting data from their economic and quality-of-life substudies at the Transcatheter Cardiovascular Therapeutics Conference in October 2015.

Karina Huynh 